\title{
An algorithm for thimble regularization of lattice field theories (and possibly not only for that)
}

\author{
Francesco Di Renzo* \\ University of Parma and INFN \\ E-mail: francesco.direnzo@unipr.it
}

\section{Giovanni Eruzzi}

Università di Parma and INFN

E-mail: giovanni.eruzzi@fis.unipr.it

\section{Michele Brambilla}

Università di Parma and INFN

E-mail: michele.brambillaefis.unipr.it

\begin{abstract}
In the context of thimble regularization of lattice field theories we are developing a new simulation algorithm. The main difficulty is to devise a sampling of configurations on a non-trivial manifold, which is defined as the hypersurface formed by the union of all paths of steepest descent of the complex action ending in a given saddle point. The main point with the new algorithm is the one-to-one correspondence of configurations and action values on a given steepest descent curve, which can in turn be seen as a steepest ascent if one changes the sign of the "time" variable. We report on the basic ideas and speculations.
\end{abstract}

The 32nd International Symposium on Lattice Field Theory

23-28 June, 2014

Columbia University New York, $N Y$

${ }^{*}$ Speaker. 


\section{Thimble regularization of lattice field theories}

The sign problem severely limits our capability to numerically solve many field theories: when the action $S$ is complex the $\exp (-S)$ factor in the functional integral can not be taken as a probability measure and standard Monte Carlo techniques can not be applied. Following a pioneering paper by Witten [1], a new approach to the solution of the sign problem was put forward in [2] via thimble regularization of field theories.

A straight way to understand the basic idea behind the method is to think of the virtues of saddle point evaluation of integrals, i.e. stationary phase and localization of important contributions. Both the latter are apparently good candidates to tackle the sign problem. As a matter of fact, these ideas have a generalization in the framework of Morse theory (to be precise, its complex version, known as Picard-Lefschetz theory). The generalization of Steepest Descent (SD) paths are known as Lefschetz thimbles $\mathscr{J}_{\sigma}$, in terms of which, under suitable (but not so strict) conditions on holomorphic functions $S(x)=S_{R}+i S_{I}$ and $O(x)$

$$
\int_{\mathscr{C}} d x O(x) \mathrm{e}^{-S(x)}=\sum_{\sigma} n_{\sigma} \int_{\mathscr{J}_{\sigma}} d z O(z) \mathrm{e}^{-S(z)}
$$

where the notation $(S, O)$ alludes to the functional integral that we have in mind (to really make contact with that we will need to take the normalizing factor $Z^{-1}$ into account as well). (1.1) has to be understood as follows:

- the greek index $\sigma$ is attached to stationary points $p_{\sigma}$ of the complex function $S(z)$, which is now a function of the complex variable $z$;

- each (stable) thimble $\mathscr{J}_{\sigma}$ is the union of all the SD paths that fall into $p_{\sigma}$ at inifinite time, i.e. the union of the solutions of

$$
\begin{aligned}
& \frac{d x}{d \tau}=-\frac{\partial S^{R}(x, y)}{\partial x} \\
& \frac{d y}{d \tau}=-\frac{\partial S^{R}(x, y)}{\partial y}
\end{aligned}
$$

satisfying $z(\tau=\infty)=x(\tau=\infty)+i y(\tau=\infty)=p_{\sigma}$;

- in the homological sense $\mathscr{C}=\sum_{\sigma} n_{\sigma} \mathscr{J}_{\sigma}$ and everything comes with the right real dimension, i.e. the thimbles have the same real dimension of the original domain of integration;

- the coefficients $n_{\sigma}$ count the intersections of the unstable thimbles $\mathscr{K}_{\sigma}$ with the original domain of integration; such unstable thimbles are essentially the union of all the Steepest Ascent paths associated to the critical points.

The fundamental property that makes thimbles a generalization of the saddle point method is that the imaginary part of the function $S$ stays constant on a thimble, so that (1.1) can be rewritten as

$$
\int_{\mathscr{C}} d x O(x) \mathrm{e}^{-S(x)}=\sum_{\sigma} n_{\sigma} \mathrm{e}^{-i S_{I}\left(p_{\sigma}\right)} \int_{\mathscr{J}_{\sigma}} d z O(z) \mathrm{e}^{-S_{R}(z)}
$$


It is now manifest that we have a probability distribution $\left(\mathrm{e}^{-S_{R}}\right)$ targeting which we can build a Monte Carlo. A couple of issues should be in principle addressed:

- how many thimbles contributes and what are the weights? While this is a fundamental issue, in the following we will not tackle it; it could be even be the case that for certain problems a single thimble contributes (a scenario that could be supported by universality arguments and thermodynamic limit); this has been proven to hold in the case of Bose gas [3];

- we have to keep in mind that a residual phase is now in place; this is the jacobian encoding the relative orientation between the canonical complex volume-form and the real volumeform, characterizing the tangent space of the thimble. A very good message with this respect is that on quite general ground this phase can be expected to be rather smooth, an expectation that has been proven to hold in [4]. Also, the residual phase can be quite efficiently computed by stochastic methods [5]. Once computed, the residual phase can be taken into account by reweighting.

A very simple, but effective playground in which all these concepts can be seen at work is the toy model which is discussed in [6], to which we will refer also in the following.

\section{Algorithmic challenges}

As a solution of the sign problem the thimble formulation looks natural; on the other side, it poses an intriguing algorithmic challenge: how can one devise a Monte Carlo that stays on the thimble? We now address the problem having in mind a (lattice) quantum field theory described by fields $\phi_{a, x}$ which upon complexification get a real $\left(\phi_{a, x}^{(R)}\right)$ and an imaginary part $\left(\phi_{a, x}^{(I)}\right) ; S_{R}$ has to be understood as the real part of the action.

\subsection{The Aurora algorithm}

Langevin is in a sense the natural candidate

$$
\begin{aligned}
\frac{d}{d \tau} \phi_{a, x}^{(R)} & =-\frac{\delta S^{R}}{\delta \phi_{a, x}^{(R)}}+\eta_{a, x}^{(R)} \\
\frac{d}{d \tau} \phi_{a, x}^{(I)} & =-\frac{\delta S^{R}}{\delta \phi_{a, x}^{(I)}}+\eta_{a, x}^{(I)}
\end{aligned}
$$

since the drift term maintains the field on the thimble by definition. The problem simply amounts to extracting a random gaussian noise field on the thimble. To be more precise, noise should be tangent to the thimble, which is a tough constraint since we lack a global characterization of the manifold. The original solution contained in [2] (the Aurora algorithm) is conceptually simple and elegant: since at the critical point we know the tangent space, we can get a properly oriented noise vector by transporting it (back and forth) to the critical point along our gradient flow, i.e. by imposing $\mathscr{L}_{\partial S_{R}}(\eta)=0 \Longleftrightarrow\left[\partial S_{R}, \eta\right]=0$. This amounts to writing evolution equations for the 

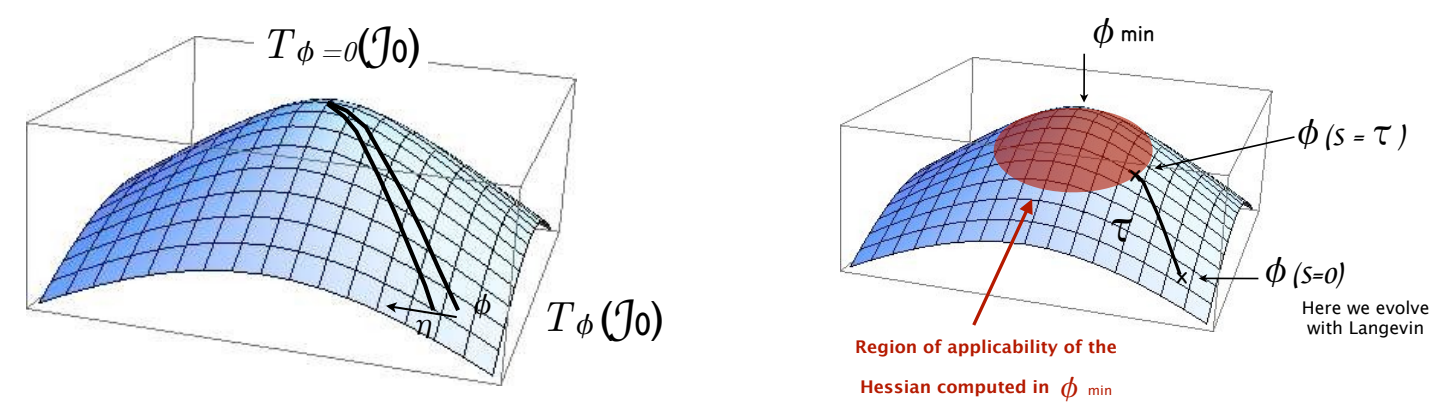

Figure 1: Aurora algorithm: transporting the noise vector back and forth to the critical point (left panel). This actually amounts (right panel) to identifying a (red) region where the tangent space is effectively that at the critical point.

noise field along the flow to have it in place at a generic point ${ }^{1}$

$$
\frac{d}{d \tau} \eta_{j}(\tau)=\sum_{k} \eta_{k}(\tau) \partial_{k} \partial_{j} S_{R}
$$

It is easy to understand that an evolution can never start from nor fall into the critical point itself: that would imply infinite evolution times. The naive question is thus: how close to the critical point is close enough? In practice one should identify a region where the tangent space is de facto aligned to the tangent space at the critical point. This is the red region in the right panel of Figure 1. In principle lucky cases can occur when the functional integral measure is peaked in a region of the manifold which is de facto in the red region. It is easy to understand that this simply asks for projecting the noise field onto the (known) tangent space at the critical point. We call this approach (whose correctness can unfortunately only be verified a posteriori) the gaussian approximation: the reader can refer to [3] for a successful application.

\subsection{Other algorithmic solutions and their problems}

An independent approach to thimble regularizaztion of lattice field theory can be found in [4]. The authors devised a Hybrid Monte Carlo algorithm; also in their case the challenge is to extract momenta tangent to the thimble, a problem that is solved by Legendre multipliers; while different from the Aurora algorithm (at least to the same extent that HMC is different from Langevin), this is quite close in spirit. In particular, it is plagued by the same difficulty: sampling in the middle of the thimble is difficult. One should add with this respect that also descending to the critical point from a generic point can be tough; what we have under fairly good control is the numerical integration of the steepest ascents from the critical point (better: from points close enough to the critical point). This can be taken as a definition of the manifold by construction; one simply changes sign to the time variable and thus to (1.2).

Another Monte Carlo algorithm on the thimble is the Metropolis that has been introduced in [7]. This works very well for toy models, but (like any Metropolis) can get frozen in the case of a field theory.

\footnotetext{
${ }^{1}$ Please notice the (quite obvious) compact notation of the noise evolution equation.
} 


\section{Ideal sampling on the thimble?}

\subsection{A lesson from a toy model}

In [6] the case of the toy model defined by $S(\phi)=\frac{1}{2} \sigma \phi^{2}+\frac{1}{4} \lambda \phi^{4}$ is discussed. Here one has to understand $\phi \in \mathbb{R}, \lambda \in \mathbb{R}^{+}, \sigma \in \mathbb{C}$. Figure 3.1 displays (left panel) the case in which $\sigma>0$ : it turns out that out of three critical points, only one (the origin) contributes, i.e. has $n_{\sigma} \neq 0$ (see [6] for details). The right panel of figure 3.1 shows how well a Langevin simulation can stay on the thimble: actually this is a trivial case, since the thimble reduces to a 1-dimensional manifold and the tangent space is simply given by the direction of the drift (i.e. by the equations of motion).
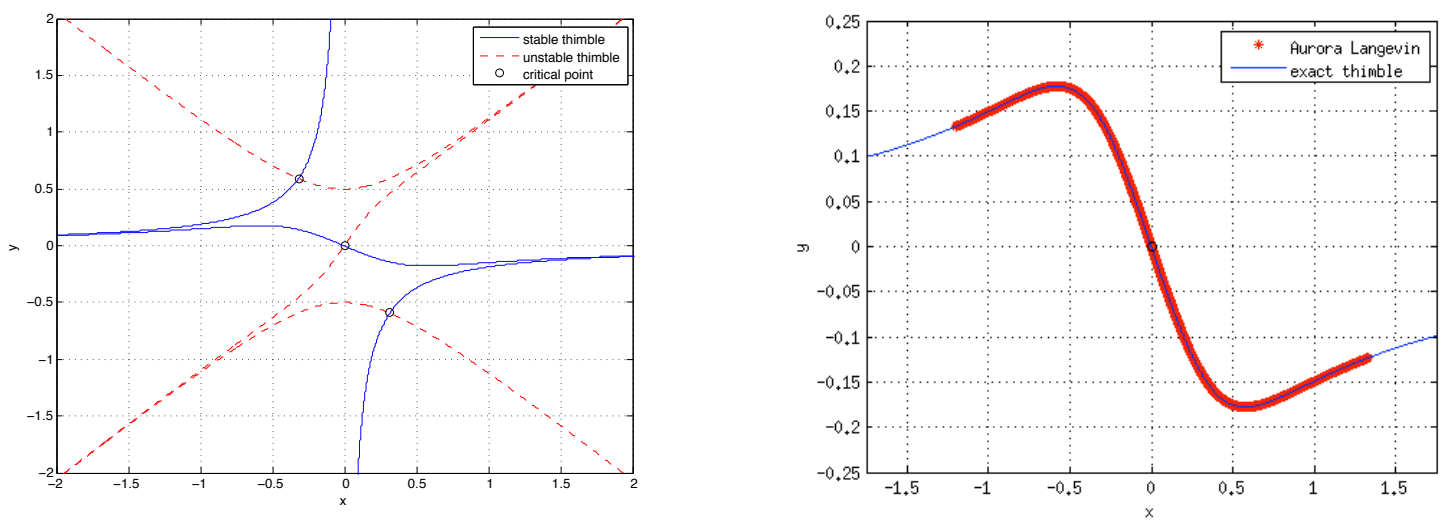

Figure 2: The $0 \mathrm{~d} \phi^{4}$ toy model in the region of the parameter space in which only one thimble out of three (left panel) contributes. Right panel: the relevant thimble covered by a Langevin simulation.

The partition function of the theory can be written as the sum of two contributions $Z=Z_{1}+Z_{2}$, each associated to one half of the thimble. Each contribution can be computed by integrating $\mathrm{e}^{-S_{R}}$ on the steepest ascent associated to an initial condition with either positive or negative component along the unique direction of the tangent space at the critical point (notice that in this case the constant phase in front of the integrals is one).

Now, on each half of the thimble there is a one-to-one correspondence action-to-field configuration. Each $Z_{i}$ is a monotonic function and can be written taking the action as the integration variable

$$
Z_{i}=\int_{S_{p_{\sigma}}}^{\infty} d S \mathrm{e}^{-S_{R}}\left|\nabla S_{R}\right|^{-1}
$$

which we can (numerically) invert to define a distribution function

$$
F_{i}\left(S^{\prime}\right)=Z_{i}^{-1} \int_{S_{p_{\sigma}}}^{S^{\prime}} d S \mathrm{e}^{-S_{R}}\left|\nabla S_{R}\right|^{-1}
$$

We are now in a position to perform ideal sampling on the thimble: simply extract a (flat) random number $\xi$ and get an action value (i.e. a field configuration) via $S=F^{-1}(\xi)$.

This ideal sampling has a couple of valuable features: 
- in order to extract configurations we only need to perform steepest ascents, i.e. the numerical integrations we have under fairly good control;

- by numerical integrating the steepest ascent paths we can perfom a heat bath sampling.

We would like to step forward from this simple setting while preserving these interesting features.

\subsection{Ideal sampling for a realistic theory?}

We now address a multi-dimensional problem. In our thimble setting, a partition function will be written (we still adhere to the notation of complex, although many, variables; in particular, $m$ is the real dimension of our manifold)

$$
Z=\int_{\mathscr{J}_{\sigma}} d z_{1} \wedge \ldots \wedge z_{m} \mathrm{e}^{-S}=\sum_{\text {charts } c} \int_{\Gamma_{c}} \prod_{i}^{m} d y_{i}^{c} \operatorname{det}(J) \mathrm{e}^{-S}=\sum_{\text {charts } c} \int_{\Gamma_{c}} \prod_{i}^{m} d y_{i}^{c} \mathrm{e}^{i \Phi} \mathrm{e}^{-S}
$$

The previous expression assumes once again that a single thimble contributes; in this context we take this as a working hypothesis, mainly for the sake of simplicity (it can be released, in principle). Apart from that, it is accurate, in particular explicitly accounting for different charts and for the residual phase (see [5] for details). We now discard the fine details (e.g. we can always account for the residual phase a posteriori) and rewrite

$$
Z=\int_{\Gamma} \prod_{i}^{m} d y_{i} \mathrm{e}^{-S}=\int \prod_{i}^{m} d n_{i} \delta\left(|\vec{n}|^{2}-1\right) Z_{\hat{n}}=\int \prod_{i}^{m} d n_{i} \delta\left(|\vec{n}|^{2}-1\right) \int d \tau \Delta_{\hat{n}}(\tau) \mathrm{e}^{-S(\hat{n}, \tau)}
$$

$\hat{n}$ is a generic direction within the tangent space at the critical point. (3.4) takes $(\hat{n}, \tau)$ as the parametrization of the generic point of the thimble. This is indeed natural: any point can be reached by leaving the critical point along a direction $\hat{n}$ and integrating steepest ascent for a time $\tau$. $Z_{\hat{n}}$ measures the contribution of the direction $\hat{n}$ to the partition function. $\Delta_{\hat{n}}(\tau)$ is in turn defined by (think of the Faddev-Popov trick)

$$
1=\Delta_{\hat{n}}(\tau) \int \prod_{j}^{m} d n_{j} \delta\left(|\vec{n}|^{2}-1\right) \int d \tau \prod_{i}^{m} \delta\left(y_{i}-y_{i}(\hat{n}, \tau)\right) .
$$

We now have all the ingredients for ideal sampling along a single steepest ascent. A probability distribution is defined for any configuration at fixed $\hat{n}$

$$
P_{\hat{n}}(\tau)=Z_{\hat{n}}^{-1} \Delta_{\hat{n}}(\tau) \mathrm{e}^{-S(\hat{n}, \tau)}
$$

so that by numerically inverting

$$
F_{\hat{n}}(\tau)=Z_{\hat{n}}^{-1} \int_{-\infty}^{\tau} d \tau^{\prime} \Delta_{\hat{n}}\left(\tau^{\prime}\right) \mathrm{e}^{-S\left(\hat{n}, \tau^{\prime}\right)}
$$

we can sample via $\tau=F_{\hat{n}}^{-1}(\xi)$, where we now parametrize the configuration with the time at which we have to stop the ascent.

(3.6) is the starting point for any attempt at ideal sampling on multi(many)-dimensional thimbles. There are of course a lot of details to fix, but this is the general setting. After the conference we have already got a few nice results for the Random Matrix Model discussed in [8]. 
One can speculate that the approach could be considered also for more general frameworks, besides thimble regularization of lattice field theories. The appealing feature is the hope that one could maybe alleviate problems connected to different topological sectors just in the same spirit of $[9]^{2}$.

\section{Conclusions}

We have put forward the basic description of an ideal sampling approach to simulating thimble regularized lattice field theories. While this has been nicely successful for toy models (see [6]) and is being proven successful for the Random Matrix Models described in [8], a lot remains to be worked out for realistic field theories.

\section{Acknowledgments}

We warmly thank L. Scorzato, M. Cristoforetti, A. Mukherjee, C. Torrero and C. Schmid for many fruitful discussions.

\section{References}

[1] E. Witten, arXiv:1001.2933 [hep-th].

[2] M. Cristoforetti et al. [AuroraScience Collaboration], Phys. Rev. D 86 (2012) 074506 [arXiv:1205.3996 [hep-lat]].

[3] M. Cristoforetti, F. Di Renzo, A. Mukherjee and L. Scorzato, Monte Carlo simulations on the Lefschetz thimble: Taming the sign problem, Phys. Rev. D 88 (2013) 5, 051501 [arXiv:1303.7204 [hep-lat]].

[4] H. Fujii, D. Honda, M. Kato, Y. Kikukawa, S. Komatsu and T. Sano, Hybrid Monte Carlo on Lefschetz thimbles - A study of the residual sign problem, JHEP 1310 (2013) 147 [arXiv:1309.4371 [hep-lat]].

[5] M. Cristoforetti, F. Di Renzo, G. Eruzzi, A. Mukherjee, C. Schmidt, L. Scorzato and C. Torrero, An efficient method to compute the residual phase on a Lefschetz thimble, Phys. Rev. D 89 (2014) 114505 [arXiv:1403.5637 [hep-lat]].

[6] G. Eruzzi and F. Di Renzo, Solution of simple toy models via thimble regularization of lattice field theory these proceedings, PoS Lattice 2014 (2014) 202.

[7] A. Mukherjee, M. Cristoforetti and L. Scorzato, Metropolis Monte Carlo integration on the Lefschetz thimble: Application to a one-plaquette model, Phys. Rev. D 88 (2013) 5, 051502 [arXiv:1308.0233 [physics.comp-ph]].

[8] A. Mollgaard and K. Splittorff, Complex Langevin Dynamics for chiral Random Matrix Theory, Phys. Rev. D 88 (2013) 116007 [arXiv:1309.4335 [hep-lat]].

[9] M. Luscher, Trivializing maps, the Wilson flow and the HMC algorithm, Commun. Math. Phys. 293 (2010) 899 [arXiv:0907.5491 [hep-lat]].

\footnotetext{
${ }^{2}$ We thank L. Del Debbio and S. Schaefer for having pointed us the relation of our approach to that of [9]
} 